Journal of Scientific Perspectives

\author{
Volume 5, Issue 1, Year 2021, pp. 55-69 \\ E - ISSN: 2587-3008 \\ URL: https://journals.gen.tr/jsp \\ DOİ: https://doi.org/10.26900/jsp.5.1.05 \\ Research Article
}

\title{
THE EFFECTS OF HIGH SALT DIET AND EXERCISE ON THE WATER-SALT BALANCE AND BLOOD PRESSURE IN RATS
}

\author{
Buket GÜNGÖR* \& Afet Seçil AKDUR** \& Coşkun SILAN*** \& \\ Hakki Engin AKSULU**** \& Orhan ŞAHIIN*****
}

* MD, Pharmacologist, MSC, Republic of Turkey Ministry of Health Antalya Provincial Directorate of Health, Department of Pharmaceutical and Medical Devices Services.

Antalya, TURKEY. e-mail: buket.gungorl@saglik.gov.tr

ORCID ID: https://orcid.org/0000-0002-5802-1635

** MD, Pharmacologist, Department of Clinical Pharmacology, Canakkale State Hospital, Turkish Ministry of Health, Canakkale, TURKEY, e-mail: shizli10@hotmail.com ORCID ID: https://orcid.org/0000-0001-5418-2442

*** MD Professor of Medical Pharmacology, Department of Pharmacology, School of Medicine, Canakkale Onsekiz Mart University, Canakkale,TURKEY, e-mail: csilan@ hotmail.com ORCID ID: https://orcid.org/0000-0002-8352-6571
**** MD Professor of Medical Pharmacology, Department of Pharmacology,
School of Medicine, Canakkale Onsekiz Mart University, Canakkale,TURKEY, e-mail: haksulu@hotmail.com ORCID ID: https://orcid.org/0000-0001-6354-4775

***** MD, Söke Fehime Faik Kocagöz State Hospital, Ministry of Health of Turkey, Aydin, Turkey, PhD Student of Toxicology, Dokuz Eylul University, School of Medicine, Department of Medical Pharmacology, Izmir, TURKEY, e-mail: sahin_2010@hotmail.com ORCID ID: https://orcid.org/0000-0003-1606-3164

Received: 30 October 2020, Accepted: 12 January 2021

\begin{abstract}
In the study which was prepared based on the factors that can take place in essential hypertension pathology; We aimed to investigate the interactions of intensive exercise, high salt and partial NOS inhibition applications with each other, the effects on water-salt balance and blood pressure, changes in the intrarenal dopaminergic system, which is an important natriuretic system, and the participation of oxidative stress. The rats were given intensive exercise on a treadmill at a speed of $25 \mathrm{~m} / \mathrm{min}$ at $5 \%$ inclination for 30 minutes a day, LNNA at a concentration of $50 \mathrm{mg} / \mathrm{L}$ and
\end{abstract}


a high salt diet of $4 \%$ for 7 days either separately or together. Blood pressures of the rats were measured on the first and last days of the experiment, and the rats were taken into metabolic cages; 24-hour water intake and urinevolume were measured. Dopamine levels were measured in 24-hour urine to detect intrarenal dopamine synthesis. In addition, oxidative stress parameters in the serums of rats; TAS, TOS and OSI levels were measured. Blood pressure was found to be high in the groups in which intensive exercise was applied together with LNNA and high salt diet. While there was no change in the water balance of this group, it was found that sodium excretion and dopamine levels increased in 24-hour urine. In addition, it was found that the total oxidant status increased in this group, and oxidative stress developed as a result of insufficient antioxidant system. It suggests that the reason of hypertension that develops with the application of intensive exercise together with LNNA and high salt diet may be due to the vascular resistance increasing effect of oxidative stress rather than water-salt retention and it points out the necessity of studies to fully detect vascular tissue oxidative stress markers and vascular oxidative damage.

Keywords: Hypertension, Intensive Exercise, Intrarenal Dopamine, Oxidative Stress

\section{INTRODUCTION}

Essential hypertension is a chronic progressive cardiovascular-kidney disease, which is manifested by constant high systemic arterial blood pressure, and whose etiopathogenesis is not fully known. Pathophysiological factors that play a role in the development of essential hypertension; excessive activity of sodium-retaining hormones and vasoconstrictor agents, insufficiency of vasodilator and natriuretic agents, increased sympathetic nervous system (SNS) activity, imbalances in renin production, high salt diet and oxidative stress (Mark et al., 1975, Carey, 2001, Touyz,2004, Chrysant, 2016).

Nitric oxide (NO) is an endogenous vasodilator agent which is released from the vascular endothelium and plays a role in the local regulation of vascular tone (Napoli and Ignarro, 2009). Also, NO, which is synthesized tonically in kidneys, plays an important role in controlling blood pressure by regulating renal hemodynamics and sodium excretion (Baylis et al., 1990, Granger and Alexander, 2000). Partial or total inhibition of NO synthesis with nitric oxide synthase (NOS) inhibitors such as L-nitro-N-arginine (LNNA) or decreased bioavailability of NO could lead to increased blood pressure (Manning et al., 1993, Vapaatalo et al., 2000, Aekthammarat et al., 2019).

High salt diet does not always lead to high blood pressure due to the balancing of natriuretic protective systems in the organism. (Titze and Luft, 2017). However, if there is renal damage due to high salt diet and an insufficiency in natriuretic systems such as intrarenal dopaminergic system, $\mathrm{NO}$, autoregulatory mechanisms cause increased peripheral vascular resistance and vascular reactivity and lead to hypertension (Shultz and Tolins, 1993, Tolins and Shultz, 1994, Yuasa et al., 2000, Banday et al., 2008, Mente, et al. 2014). The effect of the salt on blood pressure includes mixed mechanisms and these mechanisms can not be fully explained. It is suggested that high salt diet causes oxidative stress (Kopkan and Majid, 2005, Feng et al., 2017).

It is thought that stimulation of the antioxidant system in the body with regular moderate exercise prevents the formation of oxidative stress, increases NO synthesis and bioactivity, decreases peripheral vascular resistance thus contributes to the regulation of blood pressure (Kitiyakara et al., 2003). However ,increased oxygen consumption and metabolic rate with intensive, exhaustive exercise effect the mitochondrial electron transport chain, increase the catecholamine and lactic acid levels,cause temporary hypoxia and reoxygenation in some 
tissues and lead to hyperthermia. As a result, it could lead to increasing of the formation of free radicals, disruption of antioxidant-oxidant balance and oxidative stress (Alessio, 1993, Jil, 1999, Husain, 2003).

Studies have shown that development of oxidative stress, decreased nitric oxide bioavailability and hypertension occurs as a result of applying oxidant agents to test subjects. In addition to that, application of antioxidant vitamins to these test subjects with hypertension was found to increase NO bioavailability and lead blood pressure to normal levels (Banday et al., 2008, Schultz et al., 2017). Increased renal and vascular $\mathrm{O}_{2}$ cause to decreased NO bioavailability, NO mediated vasodilation disruption, decreased renal sodium reabsorption and the development of salt sensitive hypertension (Touyz,2004, Vaziri, 2000).

When salt consumption increases, with the introduction of endogenous natriuretic systems, excess salt is discharged and blood pressure remains at normal levels. In this case, intrarenal dopamine exchanges $\mathrm{Na}^{+} / \mathrm{H}^{+}$and inhibits $\mathrm{Na}^{+} \mathrm{K}^{+}$ATPase pump via D1-like receptor activation, located in the proximal tubular cells and increases urinary sodium excretion (Carey, 2001). However, in the presence of oxidative stress, D1-like receptor-G protein binding is impaired in renal proximal tubule cells. Oxidative stress causes D1 receptor dysfunction, $\mathrm{Na}^{+} \mathrm{K}^{+}$ATPase pump inhibition ability of produced dopamine is impaired, natriuresis can not occur and blood pressure increases (Majid and Kopkan, 2007, Banday et al., 2008). Oxidative stress causes increasing in blood pressure due to disruption in the activities of natriuretic and vasodilator systems.

In this study; It is aimed to investigate the effects of intensive exercise, LNNA and high salt diet individually and in combination on water-salt balance and blood pressure at a dose and time that will not increase blood pressure when administered alone.It was purposed to investigate the participation of these systems in the development of water-salt balance and hypertension by measuring parameters related to intrarenal dopamine synthesis and oxidative stress.

\section{METHODS}

Experiments were carried out on 48 male Wistar Albino rats which were 8 weeks old, weighing 190-205 gr. Rats were housed under standard conditions with a 12-hour light-dark cycle in standard cages in a room with a controlled humidity of $40 \%$ and a temperature of $22^{\circ} \mathrm{C}$. On the last day of the experiment, rats were taken to individual metabolic cages.

They had ad libitum access to food and water. For 7 days, rats were fed one of the specially prepared high salt diet with $4 \%$ salt or one of the standard rat feeds at $0.8 \%$ salt. LNNA was administered to the subjects with drinking water. Considering the amount of water consumed per day, LNNA at an average concentration of $50 \mathrm{mg} / \mathrm{L}$ was prepared daily and administered for 7 days. (The dose of LNNA applied in the experiment and the high salt diet rate were determined by pre-experiments.)

In the exercise program, "May TME 0804 Treadmill Exerciser" branded four-lane small experimental animal treadmill was used. The treadmill has an adjustable speed indicator in $0.1 \mathrm{~km} /$ hour steps and a mechanism that provides angulation between $-10^{\circ}$ and $+20^{\circ}$. The subjects who do not want to run were warned by using the electrical shock switch continuously or when desired, between 1-6 levels.

Practice exercises were not applied to the subjects. The rats were applied intensive running exercise on the treadmill at a speed of $25 \mathrm{~m} / \mathrm{min}$ and at a $5 \%$ incline for 30 minutes a day for 7 days. It has been reported that this exercise model is a intensive-consuming exercise protocol with a $\mathrm{VO}_{2 \max }>75 \%$ (Hegde and Solomon, 2015). If the subjects could not turn from 
the electric grid onto the band despite physical stimulation, they were considered exhausted and their exercises were terminated (Ji et al., 2004).

Rats were divided into eight groups with 6 rats in each group $(n=48)$. 1. Control(C )(\%0,8 salt diet and drinking water), 2. high salt group (HS) (\%4 high salt diet and drinking water), 3. Exercise group (E ) (intensive exercise; $25 \mathrm{~m} / \mathrm{min}$ speed and $\% 5$ slope for 30 minutes in a day),4. LNNA group (LNNA $50 \mathrm{mg} / \mathrm{L}$ concentration with water), 5. HS+E (\%4 high salt diet and intensive exercise), 6. E+LNNA (LNNA $50 \mathrm{mg} / \mathrm{L}$ concentration with water and intensive exercise ), 7. LNNA+ HS (LNNA $50 \mathrm{mg} / \mathrm{L}$ concentration with water and high salt diet), 8. E+LNNA+ HS (intensive exercise, LNNA $50 \mathrm{mg} / \mathrm{L}$ concentration with water and high salt diet). Systolic blood pressure measurements of rats were made by indirect tail cuff method from the tail on days 0 and 7 of the experiment (MAY BPHR 9610-PC TAIL-CUFF Indirect Blood Pressure Recorder, Ankara, Turkey). The measurements of the conscious subjects were made in a quiet laboratory environment after approximately 20 minutes of rest period, when the regular signal sound was received. The systolic blood pressure values were recorded in the computer. Systolic blood pressure averages were calculated by taking 3 measurements from each rat.

Rats were transferred to metabolic cages on the last day of the experimental protocol and the amount of water they drank and the volume of urine they produced for 24 hours were determined. At the end of the experiment, intracardiac blood was collected from rats under mild ether anesthesia and the subjects were decapitated. $\mathrm{Na}^{+}$levels in serum and urine samples were measured using an autoanalyzer (ROCHE COBAS 6000).

The water balance of the rats (volume of water intaken- volume of urine $=$ water balance) was calculated by measuring the 24 hour water intake and urine volumeof the rats taken into metabolic cages. Urine dopamine levels were measured by the Duzen Laboratories group using a chemical detector and high pressure liquid chromatography device (HPLC) from the collected 24 hour urine samples.

Serum Total Antioxidant (TAS), Serum Total Oxidant levels were measured by a fully automated method developed by Erel (Erel, 2004, Erel, 2005). The oxidative stress index was expressed as the percentage of the total oxidant status levels of the samples to the total antioxidant status of the samples. OSI was calculated as TOS $(\mu \mathrm{mol} \mathrm{H} 2 \mathrm{O} 2$ equivalent/L) / TAS ( $\mu$ mol Trolox equivalent/L)x100. In case the OSI value is greater than 1 , it was evaluated as oxidative stress. Samples were studied in Rel Assay Laboratory, Gaziantep, with colorimetric method in a fully automatic biochemistry autoanalyzer of Vital Scientific brand in accordance with the Erel Method. RL 0017 coded Rel Assay kits were used for TAS and RL 0024 coded Rel Assay kits were used for TOS.

The data obtained were expressed as mean \pm standard error (SD). Statistical differences were calculated using "independent student- $t$ " tests in independent groups. Paired student-t test was used to evaluate the difference between the values of the same group on days 0 and 7. Student's test was used in the interpretation of the obtained results and a $p$ value of $<0.05$ was considered statistically significant.

\section{RESULTS}

There was not statistically significant difference between the first and last weights of the groups.

\subsection{Blood Pressures:}

The initial blood pressure of the subjects was determined similarly. At the end of the experiment, it was determined that the blood pressure values of the groups were not affected 
by these applications when the final blood pressure values of the groups that were applied intensive exercise alone, high salt or LNNA were compared with the initial blood pressure values (Table 1).

It was found that the final blood pressure values of the groups which were applied Exercise + Salt, Exercise + LNNA and LNNA + High Salt increased significantly compared to the initial blood pressure values. In addition, it was found that the combination of intensive exercise, high salt and LNNA significantly increased blood pressure values $(\mathrm{n}=6 ; 168.38 \pm$ 4.25) compared to the other groups $(\mathrm{P}<0.05)$ (Table 1).

Table 1. Comparison of the blood pressure values of the groups measured at the beginning and at the end of the experiment.

\begin{tabular}{|l|l|l|}
\hline & First measured (mmHg) & Last measured (mmHg) \\
\hline Control (n=6) & $127,33 \pm 1,1$ & $127,70 \pm 1,1$ \\
\hline High Salt (n=6) & $128,88 \pm 1,15$ & $129,86 \pm 1,4$ \\
\hline Exercise (n=6) & $128,42 \pm 1,12$ & $134,92 \pm 2,93$ \\
\hline LNNA (n=6) & $125,48 \pm 2,32$ & $131,30 \pm 1,44$ \\
\hline Exercise + High Salt (n=6) & $128,08 \pm 0,95$ & $140,85 \pm 1,96^{* *}$ \\
\hline Exercise + LNNA (n=6) & $128,40 \pm 1,92$ & $135,45 \pm 1,79^{* *}$ \\
\hline High Salt + LNNA (n=6) & $128,53 \pm 2,41$ & $141,08 \pm 1,84^{* *}$ \\
\hline $\begin{array}{l}\text { Exercise + High Salt + } \\
\text { LNNA (n=6) }\end{array}$ & $127,17 \pm 2,01$ & $168,38 \pm 4,25^{* * *}$ \\
\hline
\end{tabular}

* Compared to control group, $\mathrm{p}<0.05$

** $\mathrm{P}<0.05$ Compared to baseline blood pressure values within the group $\quad \beta \quad \mathrm{P}<0.05$ according to the other applications groups

\subsection{Effects on Water Intake, Urine Volume and Water Balances}

On the last day of the experiment, 24 hour water intake and urinevolume of the subjects which were taken into metabolic cages were determined and their water balances were calculated.

Administration of high salt diet alone significantly increased 24 hour water intake $(\mathrm{n}=$ $6 ; 36.5 \pm 0.89)$ compared to both the control and the high salt and exercise group $(\mathrm{P}<0.05)$ (Table 2). The 24 hour urine output of this group $(n=6 ; 7.67 \pm 0.42)$ was found lower than the groups which were administered exercise and LNNA (Table 2). Only high salt application increased 24 hours water intake and decreased the volume of urine. Therefore, the water balance of high salt diet $(n=6 ; 28.8 \pm 1.14)$; increased compared to control group and the groups in which high salt administered with exercise or LNNA. LNNA application alone did not change the 24 hour water intake, but significantly reduced the volume of urine $(n=6$; $6.17 \pm 0.49)$. Only the water balance of the LNNA group $(n=6 ; 28.3 \pm 1.58)$ increased compared to the control and the groups in which LNNA was administered with salt and / or exercise, water-salt retention occurred in the body (Table 2). 
Table2. Comparison of the 24 hour water intake, urine volume and water balance data of the groups.

\begin{tabular}{|l|l|l|l|}
\hline & $\begin{array}{l}\text { Water intake } \\
(\mathbf{m l} / \text { day) }\end{array}$ & $\begin{array}{l}\text { Urine output } \\
(\mathbf{m l} / \text { day })\end{array}$ & $\begin{array}{l}\text { Water balances } \\
(\mathbf{m l} / \mathbf{d a y})\end{array}$ \\
\hline Control (n=6) & $31 \pm 1,2$ & $9,3 \pm 1,2$ & $21,6 \pm 0,3$ \\
\hline High Salt (n=6) & $36,5 \pm 0,89 * \epsilon$ & $7,67 \pm 0,42^{\epsilon * *}$ & $28,8 \pm 1,14 * * * \epsilon$ \\
\hline Exercise (n=6) & $34 \pm 2,35$ & $8 \pm 1,03$ & $26 \pm 1,9$ \\
\hline LNNA (n=6) & $34,5 \pm 1,38$ & $6,17 \pm 0,49 * \# \Omega^{* *}$ & $28,3 \pm 1,58 * \# \Omega^{* *}$ \\
\hline Exercise+ High Salt (n=6) & $30 \pm 2,24$ & $8 \pm 0,37$ & $22 \quad \pm 2,03$ \\
\hline Exercise+ LNNA (n=6) & $32,33 \pm 2,14$ & $8,01 \pm 0,6$ & $24,1 \pm 1,84$ \\
\hline High Salt + LNNA (n=6) & $30,8 \pm 2,51$ & $11,5 \pm 1,36$ & $19,33 \pm 2,17$ \\
\hline $\begin{array}{l}\text { Exercise + High Salt + } \\
\text { LNNA (n=6) }\end{array}$ & $33,67 \pm 2,07$ & $12,33 \pm 1,84$ & $22,8 \pm 1,8$ \\
\hline
\end{tabular}

* Compared to control group, $\mathrm{p}<0.05 €$ Compared to Exercise + high salt group, $\mathrm{p}<0.05$

$\Omega$ Compared to Exercise + LNNA group, $\mathrm{p}<0.05$ \# Compared to high salt + LNNA group, $\mathrm{p}<0.05$

** Compared to Exercise, high salt + LNNA group, $\mathrm{p}<0.05$

\subsection{Serum Sodium and Urine Sodium Values}

High salt, exercise and LNNA applications did not affect serum sodium values. While LNNA administration alone did not change 24 hour urinary sodium level, urinary sodium concentrations of the group in which LNNA was administered with high salt diet $(\mathrm{n}=6 ; 1.81$ \pm 0.12 ) was found higher than control group, LNNA group or high salt diet group $(\mathrm{P}<0.05)$. Simultaneously LNNA administration increased urinary sodium excretion in subjects which were given high salt diet. The 24 hours urinary sodium values of the group in which exercise, high salt diet and LNNA were applied together $(\mathrm{n}=6 ; 3.23 \pm 0.45)$ were found to be statistically significantly higher than both the control and the other groups $(\mathrm{P}<0,05)$. (Table $3)$. 
Table 3: Comparison of serum sodium values of subjects and sodium concentration in milliliter measured from 24 hour urine samples collected from subjects.

\begin{tabular}{|c|c|c|}
\hline & $\begin{array}{l}\text { Serum sodium } \\
\text { concentrations }(m E q / l)\end{array}$ & $\begin{array}{l}\text { Urine sodium concentrations } \\
(m E q / l)\end{array}$ \\
\hline Control $(n=6)$ & $144,1 \pm 0,6$ & $0,77 \pm 0,1$ \\
\hline High Salt $(n=6)$ & $143,67 \pm 0,92$ & $0,76 \pm 0,08$ \\
\hline Exercise $(n=6)$ & $143,83 \pm 0,17$ & $0,86 \pm 0,11$ \\
\hline LNNA $(n=6)$ & $143,17 \pm 0,7$ & $0,72 \pm 0,20$ \\
\hline Exercise + High Salt $(n=6)$ & $143 \pm 0,63$ & $0,78 \pm 0,08$ \\
\hline Exercise + LNNA $(n=5)$ & $144,17 \pm 0,4$ & $1,69 \pm 0,34$ \\
\hline High Salt + LNNA $(n=6)$ & $144 \pm 1$ & $1,81 \pm 0,12 * \# £$ \\
\hline $\begin{array}{l}\text { Exercise }+ \text { High Salt }+ \text { LNNA } \\
(n=6)\end{array}$ & $143,33 \pm 1,23$ & $3,23 \pm 0,45^{* \pm \# \beta \in \infty}$ \\
\hline
\end{tabular}

\subsection{Urine Dopamine Values}

The urine dopamine concentration of the high salt diet and exercise group was found to be higher than the control group. In addition, the urine dopamine concentration of this group is higher than the other groups $(\mathrm{n}=6 ; 46.4 \pm 14.8)$ however, it is not statistically significant since its standard error is high. The urine dopamine concentration $(\mathrm{n}=6 ; 35.8 \pm$ 10.2) of the group in which high salt diet, exercise and LNNA were applied together was found to be higher than the groups in which exercise and LNNA were administered separately and together and the control group $(\mathrm{P}<0,05)$ (Figure 1).

Figure 1. Comparison of 24 Hour urine Dopamine concentrations

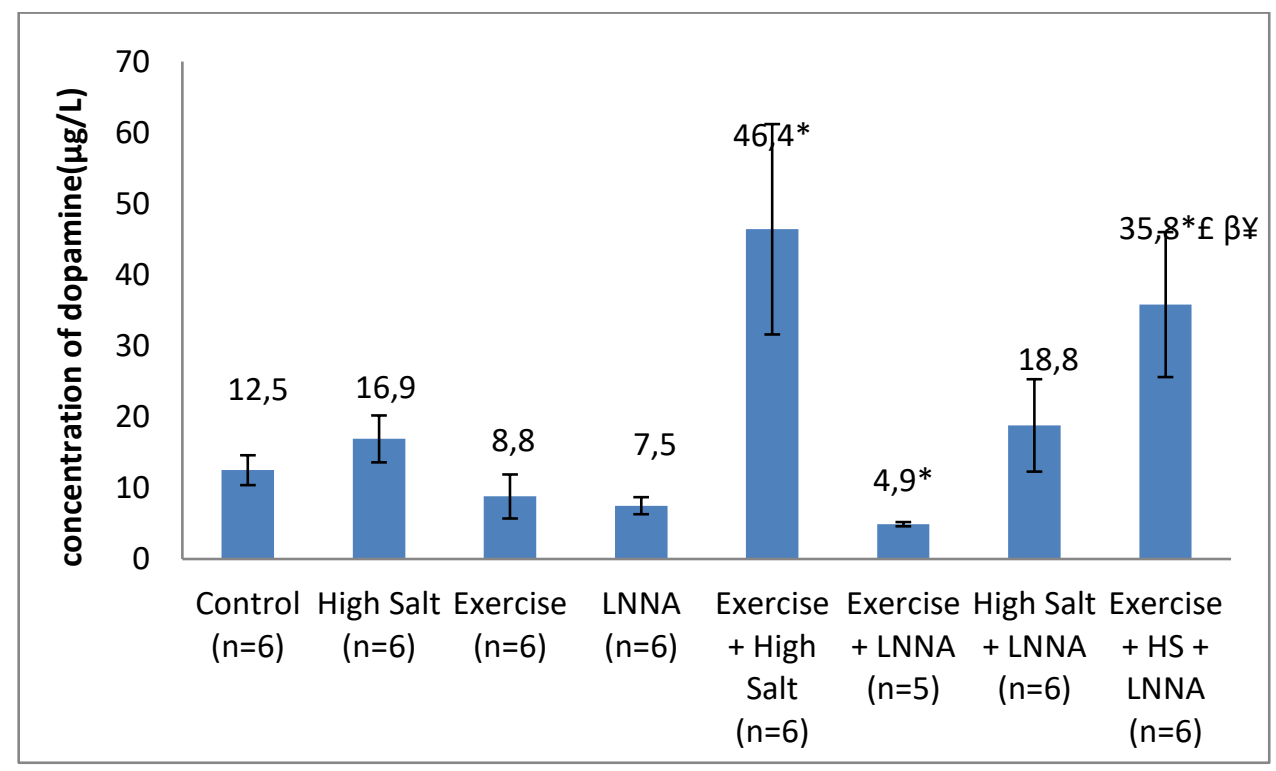

\footnotetext{
* Compared to control group, $\mathrm{p}<0.05 \quad{ }^{£}$ Compared to LNNA group, $\mathrm{p}<0.05$

${ }^{\beta}$ Compared to Exercise group, $p<0.05 ¥$ Compared to exercise + LNNA group, $p<0.05$
} 


\subsection{Serum Total Oxidant Status (TOS), Total Antioxidant Status (TAS) and Oxidative Stress Index (OSI) values}

Application of exercise and LNNA alone or together did not significantly affect serum total oxidant status values. TOS values of the group in which high salt diet was applied alone $(\mathrm{n}=6 ; 24.11 \pm 1.6)$ were found to be higher than TOS values of the control group and the groups in which high salt diet was applied with LNNA and/or exercise. In addition, serum total oxidant status values $(\mathrm{n}=6 ; 18.05 \pm 2.4)$ of the group in which high salt diet was applied with LNNA and exercise were found to be higher than the control group $(\mathrm{P}<0.05)$. Serum total antioxidant status values $(\mathrm{n}=6 ; 1.91 \pm 0.09)$ of the group in which the high salt diet $(\mathrm{n}=$ $6 ; 1.91 \pm 0.09)$ was applied alone were found to be higher than the serum total antioxidant status values of the groups in which the high salt diet was applied with LNNA and / or exercise. Other applications did not significantly affect serum total antioxidant status.

Exercise or LNNA alone did not affect the oxidative stress index (OSI) values. However, the oxidative stress index (OSI) values of the group in which exercise and LNNA $(\mathrm{n}=6 ; 1.06 \pm 0.12)$ were applied together were found to be higher than the control group. The OSI values $(\mathrm{n}=6 ; 1.27 \pm 0.09)$ of the high salt diet alone were found to be significantly higher than the control group (Table 4).

Table 4: Comparison of serum TOS = Total Oxidant Status, TAS = total antioxidant Status, OSI = oxidative stress index values of the groups.

\begin{tabular}{|l|l|l|l|}
\hline & TAS & TOS & OSI \\
\hline Control (n=6) & $1,71 \pm 0,08$ & $11,96 \pm 1,8$ & $0,64 \pm 0,09$ \\
\hline High Salt (n=6) & $1,91 \pm 0,09 \boldsymbol{\epsilon}^{* * \infty}$ & $24,11 \pm 1,6 * \epsilon_{\infty}$ & $1,27 \pm 0,09 *$ \\
\hline Exercise (n=6) & $1,41 \pm 0,06$ & $13,31 \pm 2,1$ & $0,93 \pm 0,13$ \\
\hline LNNA (n=6) & $1,52 \pm 0,06$ & $14,38 \pm 2,2$ & $0,93 \pm 0,11$ \\
\hline Exercise + High Salt (n=6) & $1,58 \pm 0,12$ & $16,03 \pm 2,1$ & $0,99 \pm 0,09 *$ \\
\hline Exercise + LNNA (n=6) & $1,47 \pm 0,05$ & $16,05 \pm 3,1$ & $1,06 \pm 0,12^{*}$ \\
\hline High Salt + LNNA (n=6) & $1,05 \pm 0,06$ & $16,06 \pm 2,4$ & $1,04 \pm 0,12^{*}$ \\
\hline $\begin{array}{l}\text { Exercise + High Salt }+ \\
\text { LNNA (n=6) }\end{array}$ & $1,61 \pm 0,07$ & $18,05 \pm 2,4^{*}$ & $1,12 \pm 0,14 *$ \\
\hline
\end{tabular}

* Compared to control group, $p<0.05 \quad$ B Compared to Exercise group, $p<0.05$

${ }^{\epsilon}$ Compared to Exercise +high salt group, $\mathrm{p}<0.05{ }^{\infty}$ Compared to high salt + LNNA group, $\mathrm{p}<0.05 \quad * *$ Compared to Exercise, high salt + LNNA group, $\mathrm{p}<0.05$

In addition, the oxidative stress index (OSI) values of the groups in which the high salt diet was administered with exercise and / or LNNA $(n=6 ; 1.12 \pm 0.14)$ were found to be significantly higher than the control group $(\mathrm{P}<0.05)$. 


\section{DISCUSSION}

Various mechanisms that are claimed to be participating in essential hypertension; it was aimed to investigate the effects on blood pressure, water-salt balance, intrarenal dopamine synthesis and oxidative stress development due to applying intensive exercise and high salt diet separately and in combination with the NO synthesis inhibitor. In this study, it was shown for the first time that LNNA, high salt diet and intense exercise have no effect on blood pressure at the dose and time when they were applied alone. However it was found that they increased the blood pressure when they were applied together. In addition, when three of these factors were applied together, the increase in blood pressure was significantly more aggravated compared when two of these factors were applied together.

NOS inhibition and exercise studies have been conducted previously, however, these practices were generally aimed at investigating the effect of regular and moderate exercise on healing hypertension (Cornelissen and Smart, 2013, Hegde and Solomon, 2015). For example, Kuru et al. found that the NOS activity of the group that was regularly exercised increased and blood pressure decreased significantly when the hypertensive group that exercised moderately and regularly was compared with the sedentary hypertensive group in hypertension which was developed with L-NAME at a dose of $25 \mathrm{mg} / \mathrm{kg}$ (Kuru et al., 2009). It is known that regular, moderate exercise reduces blood pressure in hypertension (Kitiyakara et al., 2003, Cornelissen and Smart, 2013). In contrast to regular and moderate exercise, intensive exercise causes oxidative stress. Oxidative stress is known to be a participant in the development of hypertension (Kopkan and Majid, 2005, Powers and Jackson, 2008, Schultz et al., 2017). However, the effect of intensive exercise on blood pressure is not fully known. Although intensive exercise alone is not expected to cause hypertension, these informations suggest that when it is applied together with other factors which participate to the pathogenesis of hypertension, intensive exercise may cause or aggravate hypertension. Therefore, in this study, it was predicted that oxidative stress caused by intensive exercise may contribute to the development of hypertension. Indeed, this study revealed the blood pressure increasing effect of intensive exercise, partial NOS inhibition or high salt diet. It is interesting that while LNNA or high salt diet did not increase blood pressure when they were applied alone, blood pressure was increased when intensive exercise application was added to them. When the reasons for the increase in blood pressure are questioned; parameters for water-salt balance, serum sodium concentration, urine dopamine levels and oxidative stress should be evaluated.

In this study, when the 24 hour water intake between the groups was compared, it was found that was increased in the high salt diet group and the other applications did not significantly affect water intake. In addition, when 24 hour urine volumes were compared; LNNA administration significantly reduced it. In the LNNA group, the decrease in urine volume disappeared with the addition of high salt diet. (Table 2). When the water balance table was examined, it was seen that there was a significant increase in LNNA or high salt applications alone. With LNNA application; while 24 hour water intake was not affected, a significant decrease in thevolume of urine suggests that the amount of water which was retained in the body has increased. It has been determined that high salt application increased 24 hour water intake and decreased the volume of urine, thus it increased the water balance. Increasing water balance suggests that there may be water retention in the body, but although these applications increased water balance, a significant increase in body weight did not occur.

While the water balance of both the high salt diet and LNNA group and the group to which exercise was added to these applications, did not change, the 24 hour urine volume increased. It suggests that the increased volume of urine in the groups which were applied 
high salt diet or LNNA alone may be associated with increased blood pressure when it is considered that blood pressure of these groups increased. When blood pressure increases, sodium and water excretion from the kidneys increases, fluid volume decreases and blood pressure returns to normal levels. This phenomenon is defined as the relationship of pressurenatriuresis (Johnson and Freeman, 1992, Titze and Luft, 2017). As a matter of fact, urinary sodium and water excretion of these groups also increased. Subsequent autoregulatory mechanisms cause hypertension by increasing extrarenal peripheral vascular resistance and vascular reactivity. In high salt diet, the systems that regulate the salt balance of the organism come into play, the excess salt taken is removed and the blood pressure remains at normal levels. Intrarenal dopamine plays an important role in the maintenance of blood pressure and sodium-water homeostasis, with its vasodilator and natriuretic activity (Carey, 2001, Banday and Lokhandwala, 2020). Intrarenal dopamine achieves this effect generally through renal D1-like receptors (DA-1) -G protein coupling. DA-1 receptors inhibit $\mathrm{Na}+/ \mathrm{H}+$ exchange (apical) and $\mathrm{Na}^{+} \mathrm{K}^{+}$ATPase pump (basolateral) especially in the proximal tubule and increase urinary sodium excretion. Inadequate intrarenal dopamine synthesis or activity is one of the factors that play a role in the pathogenesis of hypertension and indicator of intrarenal dopamine synthesis is dopamine levels in urine (Banday et al., 2008,a, Banday and Lokhandwala,b, 2020).

Wang et al. administered normal $(0.28 \%)$ salt or high (4\%) salt for 5 days to anesthetized rats to investigate the effectiveness of intrarenal dopamine in diuresis and natriuresis and they showed that the water-sodium excretions and urine dopamine concentrations of the high salt group increased (Wang, et al., 1997). In another study, increasing the amount of salt consumed for 8 days by $209-259 \mathrm{mEq}$ in normal individuals who receive $9 \mathrm{mEq}$ of salt per day increased the sodium and dopamine levels measured in 24 hour urine in these individuals (Wayne et al., 1974). In our study, administration of high salt (4\%) alone did not significantly increase the urinary dopamine concentration being statistically compared to the normal $(0.8 \%)$ salt applied group. Interestingly, the combination of high salt diet with LNNA and intensive exercise significantly increased dopamine levels in 24 hour urine (Figure 1). Increased urinary dopamine levels indicate increased intra renal dopamine synthesis and seem to occur with the increase in urinary sodium excretion. However, this situation could not prevent the increase in blood pressure. As intrarenal dopamine synthesis increases during high salt $(1 \%)$ intake, it causes natriuresis with $\mathrm{Na}^{+} \mathrm{K}^{+}$ATPase pump inhibition via D1 receptors and maintains blood pressure at normal levels but with high salt diet and oxidative stress with agents such as BSO (butionine sulfoximine). It has been suggested that intrarenal dopamine synthesis is not affected, but as a result of dysfunction of dopaminergic receptors, intrarenal produced dopamine loses its $\mathrm{Na}^{+} \mathrm{K}$ + ATPase pump inhibition ability and increases blood pressure by causing water-salt retention (Banday et al., 2008,a, Banday and Lokhandwala,b, 2020). In this study, sodium excretion, urinary dopamine levels and blood pressure increased with the application of intensive exercise together with LNNA and high salt diet. Although these results show that the natriuretic function of intrarenal dopamine is not impaired, the possibility of D1 receptor dysfunction that may occur with the formation of oxidative stress in this group cannot be excluded. In this study, despite the increase in dopamine synthesis, there is a possibility of D1 receptor dysfunction due to oxidative stress. However, the increase in urinary salt excretion is thought to be due to the increased blood pressure. Although the intrarenal dopaminergic system does not work, natriuresis occurs as a result of increased blood pressure. The degree of efficacy of intrarenal dopaminergic activity on natriuresis, in other words, the degree of increase and satiety of dopaminergic activity with the degree of salt loading have not been determined yet, and there is not enough information on the behavior pattern in high blood pressure. 
People with mild hypertension were exercised with a $\mathrm{VO}_{2}$ max of $40-60 \%$; It has been found that sodium excretions and urine dopamine levels increase, blood pressure decreases (Arakawa et al., 1995). In another study, salt-sensitive Dahl rats were given a $4 \%$ high salt diet for 2 weeks, followed by $8 \mathrm{~m} /$ min 60 minutes of $\mathrm{VO}_{2} \max 50 \%$ running exercise 5 days a week for 4 weeks. It was found that salt excretions and blood pressures of rats that were exercised were similar to rats that were not exercised, but rats that were applied moderate exercise had high urinary dopamine levels and that moderate exercise increased renal dopamine production (Maeda et al., 2000). On the contrary, studies performed with intensive exercise indicate that acute vigorous exercise may have negative effects on blood pressure regulation by causing oxidative stress (Witt et al., 1992, Bergholma et al., 1999). However, information on the effect of intensive exercise on urinary dopamine levels has not been found in the literature. In this study, the intensive exercise program alone did not affect the urinary dopamine levels, the combination with LNNA caused an increase in blood pressure and significantly decreased urinary dopamine levels. Since these two applications together increase sodium excretion in the urine, although it is not significant, it suggests that the increased blood pressure cannot be associated with sodium retention. In this case, the negative effects of oxidative stress formation as a result of the combination of intensive exercise and LNNA may have played a role in the increase in blood pressure. Oxidative stress has negative effects on both sodium retention and vascular tone increase. In this study, it can be thought that oxidative stress causes vasoconstriction with negative effects of $\mathrm{O}_{2}{ }^{-}$and $\mathrm{H}_{2} \mathrm{O}_{2}$ on vascular tissues, especially as a result of oxidative stress rather than its effects on sodium retention.

In intensive exercise with $\mathrm{VO}_{2}$ max higher than $75 \%$; metabolic rate, energy and oxygen consumption are increasing. Free radicals appear as byproducts of normal exercise metabolism and the production of reactive oxygen radicals (ROS) increases. Oxidative stress develops as a result of the significant increase in ROS and insufficiency of protective systems, deterioration of the prooxidant antioxidant balance (Alessio, 1993, Loperena and Harrison 2017). It has been demonstrated that intensive exercise causes increased free radical production, depletion of antioxidant agents, and disruption of endothelium dependent relaxation with in vivo studies (Bergholma et al., 1999). In this study, it was determined that high salt diet application alone significantly increased TAS, TOS and OSI levels and caused oxidative stress. While intensive exercise or LNNA application alone did not affect the measured TAS, TOS and OSI levels; combined application of them increased the oxidative stress index. In addition, the application of high salt diet with LNNA and / or intensive exercise also increased OSI values. The antioxidant system could not prevent the increase in oxidant status and oxidative stress developed (Table 4).

High salt diet causes an increase in the activity of oxidant enzymes such as NADPH oxidase in the arteries, veins and kidneys, and thus ROS production. Previous studies showed that especially when $\mathrm{O}_{2}{ }^{-}$increases, as a result of the interaction of $\mathrm{O}_{2}^{-}$with $\mathrm{NO}$, the bioactivity of NO decreases, endothelium-dependent vasodilator response is impaired, vasoconstrictor response dominates, vascular growth factors are activated and/or antioxidant enzymes are reduced and thus oxidative stress develops. (Kopkan and Majid, 2005, Wilcox, 2005, Lenda et al.,2000). It has been reported that oxidative stress is effective in the pathology of hypertension both in hypertensive animal models and humans (Champlain et al., 2004). In addition, hydrogen peroxide $\left(\mathrm{H}_{2} \mathrm{O}_{2}\right)$, which is a free radical, is also known as an endotheliumderived hyperpolarizing factor, which is important in maintaining vascular tone. It has been shown that increased ROS when oxidative stress develops causes vasoconstriction by causing vascular $\mathrm{Ca}^{+2}$ increase (Tabet et al., 2004). 


\section{CONCLUSION}

In this study, it has been shown that the combination of intensive exercise, LNNA and high salt diet, which have no effect on blood pressure at the dose and time applied alone, increases urinary sodium excretion and renal dopamine synthesis, but significantly increases blood pressure, the antioxidant system cannot prevent the increase in oxidant status, and oxidative stress develops.

The findings suggest that the reason for hypertension developed by the combination of intensive exercise, LNNA and high salt diet may be due to the vascular resistance increasing effect of oxidative stress rather than water-salt retention.

Conflict of Interest: No conflict of interest was declared by the authors.

Acknowledgments: "This work was supported by the Research Fund of the Canakkale Onsekiz Mart University; project number:2012/32 (Institutional approval number: 2012/01-12) 


\section{REFERENCES}

AEKTHAMMARAT, D., PANNANGPETCH, P., et al. (2019). Moringa oleifera leaf extract lowers high blood pressure by alleviating vascular dysfunction and decreasing oxidative stress in L-NAME hypertensive rats. Phytomedicine, 54, 9-16.

ALESSIO, H.M. (1993). Exercise-Induced Oxidative Stress. Medicine and Science in Sports and Exercise, 25, 218-224.

ARAKAWA, K., MIURA, S., et al. (1995). Activation of renal dopamine system by physicalexercise. Hypertens Res., 18, 573-577.

BANDAY, A.A. and LOKHANDWALA, M. (2020a). Renal dopamine oxidation and inflammation in high salt fed rats. Journal of the American Heart Association, 9(1), e014977.

BANDAY, A.A., LAU, Y.S., et al. (2008b). Oxidative stress causes renal dopamine d1 receptor dysfunction and salt-sensitive hypertension in sprague-dawley rats. Hypertension, 51, 367-375.

BAYLIS, C., HARTON, P., et al. (1990). Endothelial derived relaxing factor controls renal hemodynamics in the normal rat kidney. J Am Soc Nephrol, 1(6), 875-81.

BERGHOLMA, R., MÄKIMATTILAA, S., et al. (1999). Intense physical training decreases circulating antioxidants and endothelium-dependent vasodilatation in vivo. Atherosclerosis, 2, 341-349.

CAREY, R.M. (2001). Renal dopamine system: paracrine regulator of sodium homeostasis and blood pressure. Hypertension, 38, 297-302.

CHAMPLAIN, J., WU, R., et al. (2004). Oxidative stress in hypertension clinical and experimental hypertension. J Hypertens, 7-8, 593-601.

CHRYSANT, S.G. (2016). Effects of high salt intake on blood pressure and cardiovascular disease: The role of COX inhibitors. Clinical Cardiology, 39(4), 240-242.

CORNELISSEN, V.A. \& SMART, N.A. (2013). Exercise training for blood pressure: a systematic review and meta-analysis. Journal of the American heart association, 2(1), e004473.

EREL, O. (2005a). New automated colorimetric method for measuring total oxidant status. Clinical Biochemistry, 12, 1103-1111

EREL, O. (2004b). Novel automated direct measurement method for total antioxidant capacity using a new generation, more stable ABTS radical cation. Clinical Biochemistry, 4, 277-285.

FENG, W., DELL'ITALIA, L.J., et al. (2017). Novel paradigms of salt and hypertension. Journal of the American Society of Nephrology, 28.5, 1362-1369.

GRANGER, J.P. \& ALEXANDER, B.T. (2000). Abnormal pressure-natriuresis in hypertension: role of nitric oxide Acta Physiol, Scand., 168, 161-168.

HEGDE, S.M. \& SOLOMON, S.D. (2015). Influence of physical activity on hypertension and cardiac structure and function, Current hypertension reports, 17.10: 77.

HUSAIN, K. (2003). Interaction of exercise training and chronic NOS inhibition on blood pressure, heart rate, NO and antioksidants in plasma of rats. Pathophysiology, 10, 4753. 
JI, L.L, GOMEZ, M.C, et al. (2004a). Acute exercise activates nuclear factor (NF)-signaling pathway in rat skeletal muscle. FASEB J., 18, 1499-1506.

JIL, L.L. (1999). Antioxidants and oxidative stress in exercise. exp Biol Med., 3, 283-292.

JOHNSON, R.A. \& FREEMAN, R.H. (1992). Pressure natriuresis in rats during blockade of the L-arginine/nitric oxide pathway Hypertension. Acta Clinica Belgica, 19, 333-338.

KITIYAKARA, C., CHABRASHVILI, T., et al. (2003). Salt intake, oxidative stress and renal expression of NADPH oxidase and superoxide dismutase. J Am Soc Nephrol, 14(11), 2775-82.

KOPKAN, L. \& MAJID, D.S. (2005). Superoxide contributes to development of salt sensitivity and hypertension induced by nitric oxide deficiency. Hypertension, 46, 1026-31.

KURU, O, SENTURK, U.K., et al., (2009). Effect of exercise training on resistance arteries in rats with chronic NOS inhibition. J Appl Physiol, 107, 896-902.

LENDA, D.M., SAULS B., et al., (2000). Reactive oxygen species may contribute to reduced endothelium-dependent dilation in rats fed high salt. American Journal of Physiology Heart and Circulatory P. hysiology, 279, 7-14.

LOPERENA, R. \& HARRISON D.G. (2017). Oxidative stress and hypertensive diseases. Medical Clinics., 101(1), 169-193.

MAEDA, H., SASAGURI, M., et al. (2000). Roles of renal dopamine and kallikrein-kinin systems in antihypertensive mechanisms of exercise in rats. Hypertens Res., 23, 511519.

MAJID, D.S. \& KOPKAN, L. (2007). Nitric oxide and superoxide interactions in the kidney and their implication in the development of salt-sensitive hypertension. Clin Exp. Pharmacol Physiol, 34, 946-52.

MANNING, R.D., HU, L., et al. (1993). Cardiovascular responses to long-term blockade of nitric oxide synthesis. Hypertension, 22, 40-8.

MARK, A.L., LAWTON, W.J., et al. (1975). Effects of high and low sodium intake on arterial pressure and forearm vasular resistance in borderline hypertension. Circulation Research, 36, 194-198.

MENTE, A., O'DONNELL, M. J., et al. (2014). Association of urinary sodium and potassium excretion with blood pressure. New England Journal of Medicine, 371(7), 601-611.

NAPOLI, C. \& IGNARRO L.J. (2009). Nitric oxide and pathogenic mechanisms involved in the development of vascular diseases. Arch Pharm Res., 32, 1103-1108.

POWERS, S.K, \& JACKSON, M.J. (2008). Exercise-induced oxidative stress: cellular mechanisms and impact on muscle force production. Physiol Rev., 88(4), 1243-1276.

SCHULTZ, M.G, GERCHE, L., et al. (2017). Blood pressure response to exercise and cardiovascular disease. Current hypertension reports, 19.11, 89.

SHULTZ, P.J. \& TOLINS, P.J. (1993). Adaptation to increased dietary salt intake in the rat role of endogenous nitric oxide. J. Clin. Invest., 91, 642-65.

TABET, F., SAVOIA, C., et al. (2004). Differential calcium regulation by hydrogen peroxide and superoxide in vascular smooth muscle cells from SHR, $J$ Cardiovasc Pharmacol.,44, 1-9. 
TITZE, J., \& LUFT, C.F. (2017). Speculations on salt and the genesis of arterial hypertension. Kidney International, 91, 1324-1335.

TOLINS, J.P. \& SHULTZ, P.J. (1994). Endogenous nitric oxide synthesis determines sensitivity to the pressor effect of salt, Kidney Int. 46, 230-6.

TOUYZ, M.R. (2004). Reactive oxygen species, vascular oxidative stress, and redox signaling in hypertension: what is the clinical significance? Hypertension, 44, 248252 .

VAPAATALO, H., MERVAALA, E., et al. (2000). Role of endothelium and nitric oxide in experimental hypertension. Physiol Res., 49,1-10.

VAZIRI, N.D., WANG, X.Q., et al. (2000). Induction of oxidative stress by glutathione depletion causes severe hypertension in normal rats. Hypertension, 36, 142-6.

WANG, Z.Q., SIRAGY, H.M, et al., (1997). Intrarenal dopamine production and distribution in the rat: physiological control of sodium excretion. Hypertension, 29, 228-234.

WAYNE, A.R., GILL, J.R., et al. (1974). Effects of dietary sodium and of acute saline infusion o the interrelationship between dopamine excretion and adrenergic actity in man, The Journal of Clinical Investigation, 54, 194-200.

WILCOX, S.C. (2005). Oxidative stress and nitric oxide deficiency in the kidney: a critical link to hypertension? American Journal of Physiology Regulatory. Integrative and Comparative Physiology, 289, 583-597.

WITT, E.H. \& REZNICK, A.Z., et al. (1992). Exercise, Oxidative Damage and the Effects of Antioxidant Manipulation, J. Nutr., 122, 766-73.

XU, J., LI, X.X., et al. (2000). D1 receptor, gs $\alpha$, and na $+\mathrm{h}+$ exchanger interactions in the kidney in hypertension. Hypertension. 36, 395-399.

YUASA, S., LI, X., et al. (2000). Sodium sensitivity and sympathetic nervous system in hypertension induced by long-term nitric oxide blockade in rats. Clin Exp Pharmacol Physiol, 27, 18-24. 